\title{
The Role of Debate in Moving Minds
}

Management Teaching Review 2016, Vol. I(I) 19-25 (C) The Author(s) 2015

Reprints and permissions: sagepub.com/journalsPermissions.nav DOI: 10.1 I77/2379298|I562I702 mtr.sagepub.com

@SAGE

\section{Paul Donovan' and John Townsend ${ }^{2}$}

\begin{abstract}
Management development exercises aimed at passing on or scaffolding knowledge and skills in training programs are plentiful but exercises that can help change attitudes are in short supply. Role debate is a structured debating exercises where participants reconsider the way they think about important job-related issues. This article shows a case study where role debate enabled call agents to gain a greater grasp of their organization's customer service policy. In role debates, participants, working in pairs, take up opposing stances on a given issue. After a few minutes debating, participants switch roles and argue the opposite to their initial stance. This allows for issues to be considered from many perspectives and for attitudes to adjust. Role debate has a wide applicability for teachers and facilitators in numerous settings. Instructions on how to design and conduct as well as several examples of role debates are given.
\end{abstract}

\section{Keywords}

management development, training, role plays/acting, role debate, attitudinal change

\section{The Role of Debate in Apprehending Learning}

In management development and training exercises, we aim to enable learners to apprehend learning through the acquisition of knowledge, skills, and/or attitudes. In our experience, there is an abundance of tools and techniques to help us as facilitators of these events to "pass on" or to "scaffold" learning for our participants. Attitude change, or the moving of minds of learners, however, is not so well catered for. Only on rare occasions have we found useful learning approaches that are both enabling of

IMaynooth University, Kildare, Ireland

2 Mas la Papesse Cavillargues, Avignon, France

\section{Corresponding Author:}

Paul Donovan, Maynooth University, Rye Hall Extension, North Campus, Kildare, Ireland.

Email: Paul.donovan@nuim.ie 
independent and autonomous attitude change and, at the same time, are also ethical. Role Debate is one such approach.

\section{What Is Role Debate?}

Role debate is a structured debating exercise where people are encouraged to reconsider the way they do things or the way they think about certain issues. The aim is to enable participants, involved in a training or education workshop, to take the time to listen to another's point of view and, above all, to make the effort to see something from an opposing position. In a role debate exercise, pairs of participants adopt opposing positions in debating a one-sentence statement/affirmation that is related to the attitude under consideration on the training workshop. Each person prepares his or her thoughts for a few minutes initially alone and then takes 5 minutes, engaging with his or her partner, to forward arguments to first support and then disagree with the statement. At the conclusion of the debate, the workshop facilitator conducts a debriefing of the exercise to elicit the effects of the role debate on moving attitudes.

\section{Why It Works}

"A mind that is stretched by a new experience can never go back to its old dimensions" is a saying attributed to the well-known American writer Oliver Wendell Holmes Jr. In a similar way, role debate encourages our workshop participants to engage in a new experience or attitude, or to perhaps take a stance to which they are opposed. Holmes would suggest that, after such a journey, our participants are more likely to discover and adopt new perspectives on relevant topics, thus achieving a change in perspective or attitude.

Role debate operates on the dissonance normally associated with cognitive conflict. Dissonance theory suggests that if individuals take up positions that they disagree with, without extrinsic reward, then there will be attitude shift. This is because the dissonance experienced by the participant needs resolution, and without an extrinsic reward as justification, the participant shifts position to relieve the cognitive discomfort. However, role debate has none of the negative connotations of coercive or manipulative approaches. This is because such exercises contain free and informed consent, and the participant may choose not to change or to what degree to change opinion.

Some participants might be reminded of the "Flip Flop" part of the BBC comedy television program Argumental (2008-2012) during which stand-up comics improvised arguments for and against a controversial topic such as "Eating Meat Is Wrong"changing "sides" each time a buzzer sounded. Game shows in other cultures adopt similar approaches. Where participants recognize the similarity, the association with popular culture may enhance the acceptability of role debate to them.

\section{Example of Role Debate Statements}

One of our first examples of using role debate was with a service organization that wanted to train call agents in the subtleties of their customer service policy. The organization's management believed in the mantra "the customer is always right," but there 
were exceptions and nuances around the policy that the front-line call agents were finding difficult to understand. We designed and conducted a role debate exercise with the proposition "the customer is not always right" for the organization's call agents. The results were positive in that following the exercise each call agent had a more sophisticated grasp of the organization's intent regarding customer rights. This may have been possible with methods of training but we doubt that it could have been achieved as smoothly and as quickly. Examples of role debate exercises are provided in the appendix.

\section{How to Run a Role Debate Exercise}

A role debate exercise requires careful planning and organization. The facilitator should choose an appropriate debate topic that is relevant to the learning objectives of the program. The facilitator should have a reasonable level of skill in handling groups as some level of competence will be required at the debriefing stage.

A role debate should have a careful introduction to the topic and the question. The aim of this introduction is to make sure that all participants understand what is required of them. Facilitators should describe the purpose of the exercise, clearly linking it to the objectives of the session. The topic should be stated and explained briefly so that participants may link it to the context of the session.

The actual exercise should be organized as follows. Split the group into pairs.

1. Ask each partner to spend 2 to 3 minutes preparing his or her thoughts on what arguments to bring out in the 5-minute debate to follow-Partner A prepares arguments in favor of the statement and Partner B arguments against.

2. At the end of the preparation period give them 5 minutes and ask Partner A to put forward his or her arguments and ask Partner B to attempt to refute these arguments. The facilitator may want to suggest that Partner B listens to Partner A for a couple of minutes before presenting counterarguments.

3. Stop the clock and give the pairs another 2 to 3 minute to prepare new/supplementary arguments for the viewpoint OPPOSING the one they have just defended. Point out that they may not only present arguments against those which they have just presented but that they should also introduce new ones.

4. Repeat as in 2 .

Note. The more provocative the role debate sentence, the more a longer explanatory introduction may be required. One way to ensure participants' understanding of the exercise is for two facilitators to demonstrate a role debate. If this is not feasible, it may be possible to prepare a short video of such a demonstration beforehand and show it to the group.

\section{Debriefing}

Debriefing is a critical element of experiential learning without which exercises become meaningless activities. Debriefing may be described as a postexercise exploratory 
session. The purpose of debriefing is to allow participants establish connections between the exercise just completed and their world back on the job. Debriefing is thus a critical part of the learning event. When participants are enabled to reflect on their experience and relate it to the "real world" of work, they are facilitated in capturing their learning gained from the exercise. It can, therefore, form a vital part of the program.

Some of the sample debriefing questions we use are as follows:

1. What happened?

2. To what extent do you feel that you have discovered new arguments against your previously held opinions?

3. Which of those arguments did you find easy to dismiss?

4. Which of those arguments did you find more difficult to dismiss?

5. Why is this new level of understanding important?

6. How can you/we use this information back on the job?

In pairs, participants are allowed to practice answering these questions in private before they are asked to share them with the wider group. The approach prevents the participants being "put on the spot" and being rendered vulnerable to exposure in front of the group. With pairs, the facilitator enables each participant to answer the question as opposed to one person talking and everyone else listening. With pairs, everyone gets to answer every question. The sample questions "walk" the participants through a procession of the experiential event. It is also an interesting and safe way to reflect on the event.

\section{How to Write the "Debate" Statements}

Identify the issue that is the focus of the session, for example, the wearing of corporate uniforms, and the intended objective of the session also, for example, that they should be worn at all times. Create a relatively provocative, one-sentence statement/affirmation that argues AGAINST the intended objective of the session, for example, "It is unnecessary to wear the corporate uniform at all times." An effective role debate statement is one that may, at first sight, be attractive to people who have the mind-set that the organization would like to modify. However, under serious consideration, it soon becomes obviously irresponsible or illogical to defend within the organization.

Example. A manufacturer of reputedly high-quality luxury goods asked its sales teams to debate "The quality does NOT justify the price." The reason for choosing this method of attitude review was that the company realized that many members of the general public (and in some cases even employees) were unaware of the intrinsically high quality of raw materials and of the resulting high production costs and wanted the point-of-sale staff to be able to talk confidently when faced with customer spending reticence. The company even feared that some people believed that prices were artificially inflated to take advantage of the market's gullibility/snobbish need to boast about price. 
Table I. Responses to Unexpected Comments.

\begin{tabular}{ll}
\hline Objection & Our response \\
\hline $\begin{array}{l}\text { I still think that if you want a job done } \\
\text { well, you should do it yourself. }\end{array}$ & $\begin{array}{c}\text { How will you ever get promoted if there is } \\
\text { nobody to replace you? }\end{array}$ \\
$\begin{array}{l}\text { I still feel that results are more important } \\
\text { than how you get them. }\end{array}$ & $\begin{array}{c}\text { How far would you go for a manager who } \\
\text { thought that you were expendable? }\end{array}$ \\
I still feel that enforcing the corporate & On your next flight, how would you like to \\
uniform is wrong. & see your pilot wearing jeans and a tie dyed \\
& T-shirt? \\
I will still bring home stationery from the & What example does that give to those \\
office to use at home. & around you? Staff? Family? Friends? \\
I will still check my Facebook page at & How would you feel if your employees \\
work in future. & "goofed off" while on the clock? \\
Rules are meant to be broken. I can't & How will you respond following a critical \\
waste time following the handbook. & incident? \\
It is vital for me to know everything that & How will you deal with a lack of initiative in \\
my team is doing every minute of the & times of future crisis? \\
working day. I cannot use a "hands off" & \\
management style. &
\end{tabular}

To build on the explanation above, an argument that the quality does not justify the price might sound reasonable from the point of view of someone who usually buys these kinds of goods from a supermarket for purely utilitarian purposes. However, this argument will soon become obviously untenable when presented to someone who knows the background cost data, understands customer motivation, and who has agreed to work for the company!

\section{When the Unexpected Crops Up}

In a supportive learning environment, people take more risks and are prepared to reconsider long-held views; however, occasionally, some people do cling on to hardened ideas even after the role debate exercise. We have often responded to them in the Socratic ways shown in Table 1.

Note. When trainers are learning about role debate they often express concern about writing negative debate sentences, fearing that they might give people "ideas" they would not otherwise have had. Some contend that this kind of sentence will only add fuel to the flames of those existing negative participant attitudes that they are trying to change in the workshop. However, experience as well as research shows us that an argument will be all the more credible when the cons as well as the pros are examined. The obvious answer to a trainer's fears that a participant may be confirmed in his/her negative attitude regarding quality and price at the end of the exercise is, "If this is the case-better now than later!" 


\section{Appendix}

\section{Examples of Role Debate Exercises}

Example I. A role debate statement that could be used in a customer service workshop for public service employees:

In the public service sector it is important that employees who are in contact with members of the public concentrate, in their interactions, on technical issues rather than waste time on social chit chat.

Example 2. Here is one that may help newly appointed managers let go of the reins:

It is vital to know everything that your team is doing every minute of the working day.

Example 3. Similar to Example 2, this one may help with managers who may miss the point of why they have been appointed:

Managers need to know how to do every task that their employees do.

Example 4. One to help people look at the difference between managers and specialists:

Experts make good managers.

Example 5. When introducing situational leadership:

In terms of management style, the days of telling people what to do are gone.

Example 6. If fears of "Machiavellian" management practices arise:

Results are more important than how they are achieved.

Example 7. Here is a way of opening discussions about cultural diversity in the workplace:

Religious symbols must not be worn in the workplace.

Example 8. One for programs dealing with bullying and harassment (and perhaps the display of contentious flags and emblems):

My right to free speech is more important than your right not to be offended.

Example 9. This one would help employees vent feelings about having to adhere to dress codes: 
The corporate uniform is optional in non-customer facing situations.

Example 10. If petty theft/pilfering is occurring:

Taking home a paper clip from the office is not stealing.

Example II. A way of helping employees to self-discipline ... or letting them know it is $\mathrm{OK} ! !$

It's OK to check your Facebook page while at work.

Example 12. A program to introduce discernment at the front line:

Rules are meant to be broken.

\section{Declaration of Conflicting Interests}

The author(s) declared no potential conflicts of interest with respect to the research, authorship, and/or publication of this article.

\section{Funding}

The author(s) received no financial support for the research, authorship, and/or publication of this article. 